CARPATHIAN J. MATH.

Volume 37 (2021), No. 2,

Pages $311-323$
Online version at https : //www . carpathian. cunbm . utcluj. ro/

Print Edition: ISSN 1584 - 2851; Online Edition: ISSN 1843 - 4401

DOI: https://doi.org/10.37193/CJM.2021.02.16

Dedicated to Prof. Ioan A. Rus on the occasion of his $85^{\text {th }}$ anniversary

\title{
Fixed points of Osilike-Berinde- $G$-nonexpansive mappings in metric spaces endowed with graphs
}

\author{
A. KAEWKHAO ${ }^{1,2}$, C. KLANGPRAPHAN ${ }^{1,2}$ and B. PANYANAK ${ }^{1,2}$
}

\begin{abstract}
In this paper, we introduce the notion of Osilike-Berinde- $G$-nonexpansive mappings in metric spaces and show that every Osilike-Berinde- $G$-nonexpansive mapping with nonempty fixed point set is a $G$-quasinonexpansive mapping. We also prove the demiclosed principle and apply it to obtain a fixed point theorem for Osilike-Berinde- $G$-nonexpansive mappings. Strong and $\Delta$-convergence theorems of the Ishikawa iteration process for $G$-quasinonexpansive mappings are also discussed.
\end{abstract}

\section{INTRODUCTION}

Let $C$ be a nonempty subset of a metric space $(X, d)$. A mapping $f: C \rightarrow C$ is called a contraction if there exists $\lambda \in[0,1)$ such that

$$
d(f(x), f(y)) \leq \lambda d(x, y) \text { for all } x, y \in C .
$$

If (1.1) is valid when $\lambda=1$, then $T$ is said to be nonexpansive. A point $x$ in $C$ is called a fixed point of $f$ if $f(x)=x$.

Fixed point theory is an important tool for finding solutions of problems in the form of equations or inequalities. One of the fundamental and celebrated results in metric fixed point theory is the Banach contraction principle which stated that every contraction on a complete metric space always has a unique fixed point. This principle has been generalized in many directions, see, e.g., [11, 12, 13, 21, 27, 29] and references therein. Among other things, Osilike [22] generalized the concept of contractions to the following class of mappings : there exist $\lambda \in[0,1)$ and $L \in[0, \infty)$ such that

$$
d(f(x), f(y)) \leq \lambda d(x, y)+L \cdot d(x, f(x)) \text { for all } x, y \in C .
$$

In 2007, Berinde and Berinde [4] extended this concept to multi-valued mappings in the following manner : a multi-valued mapping $T: C \rightarrow C B(C)$ is called a weak contraction if there exist $\lambda \in[0,1)$ and $L \in[0, \infty)$ such that

$$
H(T(x), T(y)) \leq \lambda d(x, y)+L \cdot \operatorname{dist}(x, T(x)) \text { for all } x, y \in C .
$$

If (1.2) is valid when $\lambda=1$, then $T$ is called an Osilike-Berinde-nonexpansive mapping. A point $x$ in $C$ is a fixed point of $T$ if $x \in T(x)$. We denote by $F(T)$ the set of all fixed points of $T$.

In 2019, Bunlue and Suantai [6] proved the existence of fixed points as well as the demiclosed principle for Osilike-Berinde-nonexpansive mappings in Banach spaces satisfying the Opial's condition. It was quickly noted by Klangpraphan and Panyanak [17] that the results in [6] can be extended to complete CAT(0) spaces.

Received: 17.11.2020. In revised form: 24.05.2021. Accepted: 31.05.2021

2010 Mathematics Subject Classification. 47H09, 47H10.

Key words and phrases. Fixed point, Osilike-Berinde- $G$-nonexpansive mapping, $G$-quasinonexpansive mapping, Ishikawa iteration, uniformly convex hyperbolic space.

Corresponding author: B. Panyanak; bancha.p@cmu.ac.th 
On the other hand, Jachymski [14] combined the concepts of fixed point theory and graph theory to prove a generalization of the Banach contraction principle in a complete metric space endowed with a graph. In 2010, Beg et al. [3] extended Jachymski's result to the general setting of a multi-valued $G$-contraction. Later on, Alfuraidan and Khamsi [1] introduced the notion of multi-valued $G$-nonexpansive mappings and proved the existence of fixed points for such kind of mappings in hyperbolic metric spaces. Since then, the fixed point results in several kinds of metric spaces endowed with graphs have been developed and many papers have appeared, see, e.g., $[2,5,7,10,23,26,32,35,36,38]$.

In this paper, motivated by the ideas of [4], [14] and [22], we introduce the class of Osilike-Berinde- $G$-nonexpansive mappings in metric spaces and show that it is different from the class of Osilike-Berinde-nonexpansive mappings. We also give sufficient conditions for the existence of fixed points for Osilike-Berinde- $G$-nonexpansive mappings in uniformly convex hyperbolic spaces endowed with graphs. Moreover, we also prove strong and $\Delta$-convergence theorems of the Ishikawa iteration process for the class of $G$ quasinonexpansive mappings which includes the class of Osilike-Berinde- $G$-nonexpansive mappings as well. Our results extend and improve the results in [6, 17, 28, 31, 35] and many others.

\section{PRELIMINARIES}

Throughout this paper, $\mathbb{N}$ stands for the set of natural numbers and $\mathbb{R}$ stands for the set of real numbers. Let $G$ be a directed graph with a set of vertices $V(G)$ and a set of edges $E(G)$. In this paper, we assume that $G$ contains all loops and has no parallel edges. Let $x, y \in V(G)$. We say that $x$ dominates $y$ if $(x, y) \in E(G)$. Let $A$ and $B$ be nonempty subsets of $V(G)$. We say that $A$ dominates $B$ if $(a, b) \in E(G)$ for all $a \in A$ and $b \in B$.

Let $(X, d)$ be a metric space, $C$ a nonempty subset of $X$ and $G=(V(G), E(G))$ a directed graph such that $V(G) \subseteq C$. We denote by $C B(C)$ the family of nonempty closed bounded subsets of $C$ and by $K(C)$ the family of nonempty compact subsets of $C$. The distance from a point $x$ in $X$ to a nonempty subset $B$ of $X$ is defined by

$$
\operatorname{dist}(x, B):=\inf \{d(x, b): b \in B\} \text {. }
$$

The Pompeiu-Hausdorff distance on $C B(C)$ is defined by

$$
H(A, B):=\max \left\{\sup _{a \in A} \operatorname{dist}(a, B), \sup _{b \in B} \operatorname{dist}(b, A)\right\} \text { for all } A, B \in C B(C) .
$$

A multi-valued mapping $T: C \rightarrow C B(C)$ is said to be edge-preserving if for each $(x, y) \in$ $E(G)$, the following implication holds:

$$
u \in T(x), v \in T(y) \Longrightarrow(u, v) \in E(G) .
$$

Let $\lambda \in[0,1)$ and $L \geq 0$. The mapping $T$ is said to be $(\lambda, L)-G$-contraction if it is edgepreserving and

$$
H(T(x), T(y)) \leq \lambda d(x, y)+L \cdot \operatorname{dist}(y, T(x)) \text { for all }(x, y) \in E(G) .
$$

The existence of fixed points for $(\lambda, L)-G$-contractions is guaranteed by Tiammee and Suantai [34] in the following result.

Theorem 2.1. ([34]) Let $C$ be a nonempty closed subset of a complete metric space $(X, d)$ and $G=(V(G), E(G))$ be a directed graph such that $V(G)=C$. Let $T: C \rightarrow C B(C)$ be a $(\lambda, L)$ $G$-contraction such that $C_{T}:=\{x \in C:(x, y) \in E(G)$ for some $y \in T(x)\} \neq \emptyset$. Suppose that the following property holds:

$(*)$ for any sequence $\left\{x_{n}\right\}$ in $C$, if $x_{n} \rightarrow x$ and $\left(x_{n}, x_{n+1}\right) \in E(G)$ for all $n \in \mathbb{N}$, then there exists a subsequence $\left\{x_{n_{k}}\right\}$ of $\left\{x_{n}\right\}$ such that $\left(x_{n_{k}}, x\right) \in E(G)$ for all $k \in \mathbb{N}$. 
Then $T$ has a fixed point in $C$.

Definition 2.1. Let $(X, d)$ be a metric space, $C$ a nonempty subset of $X$ and $G=(V(G), E(G))$ a directed graph such that $V(G) \subseteq C$. A multi-valued mapping $T: C \rightarrow C B(C)$ is said to be

(i) Osilike-Berinde-nonexpansive if there exists $L \geq 0$ such that

$$
H(T(x), T(y)) \leq d(x, y)+L \cdot \operatorname{dist}(x, T(x)) \text { for all } x, y \in C ;
$$

(ii) Osilike-Berinde- $G$-nonexpansive if $T$ is edge-preserving and there exists $L \geq 0$ such that

$$
H(T(x), T(y)) \leq d(x, y)+L \cdot \operatorname{dist}(x, T(x)) \text { for all }(x, y) \in E(G)
$$

(iii) quasinonexpansive if $F(T) \neq \emptyset$ and

$$
H(T(x), T(y)) \leq d(x, y) \text { for all } x \in C \text { and } y \in F(T)
$$

(iv) $G$-quasinonexpansive if $T$ is edge-preserving and $F(T) \neq \emptyset$ and

$$
H(T(x), T(y)) \leq d(x, y) \text { for all }(x, y) \in E(G) \text { with } y \in F(T) .
$$

The following examples show that the class of Osilike-Berinde-nonexpansive mappings and the class of Osilike-Berinde- $G$-nonexpansive mappings are different.

Example 2.1. Let $X$ be the Euclidean space $\mathbb{R}^{2}$ and $C=[0,1] \times[0,1]$ and let $G=(V(G), E(G))$ be such that $V(G)=\{(0,0),(1,0)\}$ and

$$
E(G)=\{((0,0),(0,0)),((0,0),(1,0)),((1,0),(1,0))\} .
$$

The graph $G$ can be explained by the following diagram:

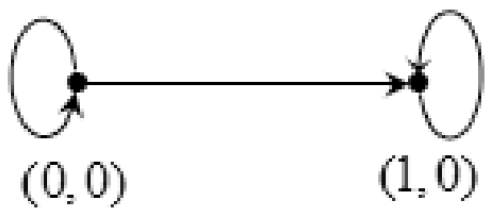

Let $T: C \rightarrow C B(C)$ be defined by

$$
T(a, b)=\{(a, 1-b)\} \text { for all }(a, b) \in C .
$$

It follows from Example 2.1 of [26] that $T$ is nonexpansive and hence Osilike-Berindenonexpansive. However, if we choose $x=(0,0), y=(1,0), u=(0,1)$ and $v=(1,1)$, then $(x, y) \in E(G), u \in T(x)$ and $v \in T(y)$. But $(u, v) \notin E(G)$. This shows that $T$ is not edge-preserving and hence is not Osilike-Berinde- $G$-nonexpansive.

Example 2.2. Let $X=\mathbb{R}, C=[0,1], G=(V(G), E(G))$ be such that $V(G)=\left[0, \frac{1}{2}\right]$ and $E(G)=\{(x, y): x, y \in V(G)\}$. Let $T: C \rightarrow C B(C)$ be defined by

$$
T(x)=\left[0, x^{2}\right] \text { for all } x \in C .
$$


It is easy to see that $T$ is edge-preserving. Let $(x, y) \in E(G)$. Then $0 \leq x, y \leq \frac{1}{2}$. Thus,

$$
H(T(x), T(y))=H\left(\left[0, x^{2}\right],\left[0, y^{2}\right]\right)=\left|x^{2}-y^{2}\right| \leq|x-y|+\operatorname{dist}(x, T(x)) .
$$

This shows that $T$ is an Osilike-Berinde- $G$-nonexpansive mapping with $L=1$. On the other hand, if $x=1$ and $y=\frac{1}{2}$, then

$$
H(T(x), T(y))=H\left([0,1],\left[0, \frac{1}{4}\right]\right)=\left|1-\frac{1}{4}\right|>\left|1-\frac{1}{2}\right|=|x-y|+L \cdot \operatorname{dist}(x, T(x)),
$$

for all $L \geq 0$. This implies that $T$ is not Osilike-Berinde-nonexpansive.

However, these two classes of mappings are identical under some additional conditions.

Proposition 2.1. Let $C$ be a nonempty subset of a metric space and $T: C \rightarrow C B(C)$ a multivalued mapping. Let $G=(V(G), E(G))$ be a directed graph such that $V(G)=C$ and $E(G)=$ $C \times C$. Then the following statements hold:

(i) $T$ is Osilike-Berinde-nonexpansive if and only if $T$ is Osilike-Berinde-G-nonexpansive.

(ii) $T$ is quasinonexpansive if and only if $T$ is $G$-quasinonexpansive.

Proof. (i) Suppose that $T$ is Osilike-Berinde-nonexpansive. Let $(x, y) \in E(G), u \in T(x)$ and $v \in T(y)$. Since $E(G)=C \times C,(u, v) \in E(G)$. This shows that $T$ is edge-preserving and hence Osilike-Berinde- $G$-nonexpansive. Conversely, suppose that $T$ is an OsilikeBerinde- $G$-nonexpansive mapping with $L \geq 0$ and given $x, y \in C$. Since $E(G)=C \times C$, $(x, y) \in E(G)$ and hence

$$
H(T(x), T(y)) \leq d(x, y)+L \cdot \operatorname{dist}(x, T(x)) .
$$

This shows that $T$ is Osilike-Berinde-nonexpansive.

The proof of (ii) is similar to (i).

The following proposition is an immediate consequence of Definition 2.1.

Proposition 2.2. The following statements hold:

(i) If $T$ is Osilike-Berinde-nonexpansive and $F(T) \neq \emptyset$, then $T$ is quasinonexpansive.

(ii) If $T$ is Osilike-Berinde-G-nonexpansive and $F(T) \neq \emptyset$, then $T$ is $G$-quasinonexpansive. hold.

The following example shows that the converses of (i) and (ii) in Proposition 2.2 do not

Example 2.3. Let $X=\mathbb{R}, C=[0,1], G=(V(G), E(G))$ be such that $V(G)=C$ and $E(G)=\{(x, y): x, y \in V(G)\}$. Let $T: C \rightarrow C B(C)$ be defined by

$$
T(x)= \begin{cases}{\left[0,\left|\frac{x}{1+x} \sin \left(\frac{1}{x}\right)\right|\right]} & \text { if } x \neq 0 \\ \{0\} & \text { if } x=0\end{cases}
$$

It is easy to see that $F(T)=\{0\}$. For $x \in(0,1]$, we have

$$
H(T(x), T(0))=\left|\frac{x}{1+x} \sin \left(\frac{1}{x}\right)\right| \leq\left|\frac{x}{1+x}\right| \leq|x-0| .
$$


This implies that $T$ is quasinonexpansive. On the other hand, for each $n \in \mathbb{N}$, we set $x_{n}:=\frac{1}{2 \pi n+\pi / 2}$ and $y_{n}:=\frac{1}{2 \pi n}$. Then

$$
\begin{aligned}
\frac{H\left(T\left(x_{n}\right), T\left(y_{n}\right)\right)-\left|x_{n}-y_{n}\right|}{\operatorname{dist}\left(x_{n}, T\left(x_{n}\right)\right)} & =\left[\frac{x_{n}}{1+x_{n}}-\left(y_{n}-x_{n}\right)\right]\left(\frac{1+x_{n}}{x_{n}^{2}}\right) \\
& =\frac{1}{x_{n}}-\frac{\left(y_{n}-x_{n}\right)\left(1+x_{n}\right)}{x_{n}^{2}} \\
& =(2 \pi n+\pi / 2)-\frac{(2 \pi n+\pi / 2+1)}{4 n} \rightarrow \infty .
\end{aligned}
$$

This implies that $T$ is not Osilike-Berinde-nonexpansive. Since $V(G)=C$ and $E(G)=C \times$ $C$, by Proposition 2.1 $T$ is $G$-quasinonexpansive and is not Osilike-Berinde- $G$-nonexpansive.

The concept of uniformly convex hyperbolic spaces is introduced by Leuştean [19].

Definition 2.2. A hyperbolic space is a metric space $(X, d)$ together with a function $W$ : $X \times X \times[0,1] \rightarrow X$ such that for all $x, y, z, w \in X$ and $t, s \in[0,1]$, we have

(W1) $d(z, W(x, y, t)) \leq(1-t) d(z, x)+t d(z, y)$;

(W2) $d(W(x, y, t), W(x, y, s))=|t-s| d(x, y)$;

(W3) $W(x, y, t)=W(y, x, 1-t)$;

(W4) $d(W(x, z, t), W(y, w, t)) \leq(1-t) d(x, y)+t d(z, w)$.

For convenience, from now on, we will replace $W(x, y, t)$ by $(1-t) x \oplus t y$. A nonempty subset $C$ of $X$ is said to be convex if $(1-t) x \oplus t y \in C$ for all $x, y \in C$ and $t \in[0,1]$. Let $G=(V(G), E(G))$ be a directed graph such that $V(G) \subseteq C$. We say that $G$ is convex if for each $x, y, u, v \in C$ and $t \in[0,1]$ such that $(x, u)$ and $(y, v)$ are in $E(G)$, we have $((1-t) x \oplus t y,(1-t) u \oplus t v) \in E(G)$. The hyperbolic space $(X, d)$ is said to be uniformly convex if for each $(r, \varepsilon) \in(0, \infty) \times(0,2]$, there exists $\delta \in(0,1]$ such that

$$
d\left(\frac{1}{2} x \oplus \frac{1}{2} y, z\right) \leq(1-\delta) r
$$

for all $x, y, z \in X$ with $d(x, z) \leq r, d(y, z) \leq r$ and $d(x, y) \geq r \varepsilon$.

A function $\eta:(0, \infty) \times(0,2] \rightarrow(0,1]$ providing such a $\delta:=\eta(r, \varepsilon)$ is called a modulus of uniform convexity. In particular, if $\eta$ is a nonincreasing function of $r$ for every fixed $\varepsilon$, then we call $\eta$ a monotone modulus of uniform convexity.

The concept of 2-uniformly convex hyperbolic spaces is introduced by Khamsi and Khan [15].

Definition 2.3. Let $(X, d)$ be a uniformly convex hyperbolic space. For each $r \in(0, \infty)$ and $\varepsilon \in(0,2]$, we define

$$
\Psi(r, \varepsilon):=\inf \left\{\frac{1}{2} d^{2}(x, z)+\frac{1}{2} d^{2}(y, z)-d^{2}\left(\frac{1}{2} x \oplus \frac{1}{2} y, z\right)\right\},
$$

where the infimum is taken over all $x, y, z \in X$ such that $d(x, z) \leq r, d(y, z) \leq r$, and $d(x, y) \geq r \varepsilon$. We say that $(X, d)$ is 2-uniformly convex if

$$
c_{M}:=\inf \left\{\frac{\Psi(r, \varepsilon)}{r^{2} \varepsilon^{2}}: r \in(0, \infty), \varepsilon \in(0,2]\right\}>0 .
$$

In [18], the authors prove that

$$
d^{2}((1-t) x \oplus t y, z) \leq(1-t) d^{2}(x, z)+t d^{2}(y, z)-4 c_{M} t(1-t) d^{2}(x, y),
$$

for all $x, y, z \in X$ and $t \in[0,1]$. 
Example 2.4. (1) Every uniformly convex Banach space is a 2-uniformly convex hyperbolic space (see [37]).

(2) If $X$ is a CAT(0) space, then it is a 2-uniformly convex hyperbolic space with $c_{M}=\frac{1}{4}$ (see [15]).

(3) If $\kappa>0$ and $X$ is a $\operatorname{CAT}(\kappa)$ space with $\operatorname{diam}(X) \leq \frac{\pi / 2-\varepsilon}{\sqrt{\kappa}}$ for some $\varepsilon \in(0, \pi / 2)$, then by Lemma 2.3 of [25] we can conclude that

$$
\Psi(r, \varepsilon)=\frac{r^{2} \varepsilon^{2} R}{8},
$$

where $R=(\pi-2 \varepsilon) \tan (\varepsilon)$. This clearly implies that $X$ is a 2-uniformly convex hyperbolic space with $c_{M}=\frac{R}{8}$.

From now on, $X$ stands for a complete 2-uniformly convex hyperbolic space with a monotone modulus of uniform convexity. Let $C$ be a nonempty subset of $X$ and $\left\{x_{n}\right\}$ be a bounded sequence in $X$. The asymptotic radius of $\left\{x_{n}\right\}$ relative to $C$ is defined by

$$
r\left(C,\left\{x_{n}\right\}\right):=\inf \left\{\limsup _{n \rightarrow \infty} d\left(x_{n}, x\right): x \in C\right\} .
$$

The asymptotic center of $\left\{x_{n}\right\}$ relative to $C$ is the set

$$
A\left(C,\left\{x_{n}\right\}\right):=\left\{x \in C: \limsup _{n \rightarrow \infty} d\left(x_{n}, x\right)=r\left(C,\left\{x_{n}\right\}\right)\right\} .
$$

It is known from [20] that if $C$ is a nonempty closed convex subset of $X$, then $A\left(C,\left\{x_{n}\right\}\right)$ consists of exactly one point.

Now, we give the concept of $\Delta$-convergence.

Definition 2.4. Let $C$ be a nonempty closed convex subset of $X$ and $x \in C$. Let $\left\{x_{n}\right\}$ be a bounded sequence in $X$. We say that $\left\{x_{n}\right\} \Delta$-converges to $x$ if $A\left(C,\left\{u_{n}\right\}\right)=\{x\}$ for every subsequence $\left\{u_{n}\right\}$ of $\left\{x_{n}\right\}$. In this case we write $\Delta-\lim _{n \rightarrow \infty} x_{n}=x$.

It is known from [16] that every bounded sequence in $X$ has a $\Delta$-convergent subsequence. The following fact can be found in [9].

Lemma 2.1. Let $C$ be a nonempty closed convex subset of $X$ and $\left\{x_{n}\right\}$ be a bounded sequence in $X$. If $A\left(C,\left\{x_{n}\right\}\right)=\{x\}$ and $\left\{u_{n}\right\}$ is a subsequence of $\left\{x_{n}\right\}$ with $A\left(C,\left\{u_{n}\right\}\right)=\{u\}$ and the sequence $\left\{d\left(x_{n}, u\right)\right\}$ converges, then $x=u$.

In [33], Tiammee et al. introduce a property that is stronger than the condition $(*)$ in Theorem 2.1.

Definition 2.5. Let $C$ be a nonempty closed convex subset of $X$ and $G=(V(G), E(G))$ be a directed graph such that $V(G)=C$. Then $C$ is said to have property $G$ if for any sequence $\left\{x_{n}\right\}$ in $C$ such that $\Delta-\lim _{n \rightarrow \infty} x_{n}=x \in C$, there exists a subsequence $\left\{x_{n_{k}}\right\}$ of $\left\{x_{n}\right\}$ such that $\left(x_{n_{k}}, x\right) \in E(G)$ for all $k \in \mathbb{N}$.

\section{FIXED POINT THEOREMS}

Let $C$ be a nonempty closed convex subset of $X$ and $I: C \rightarrow C$ be the identity mapping and $T: C \rightarrow C B(C)$ be a multi-valued mapping. We say that $I-T$ is demiclosed if for any sequence $\left\{x_{n}\right\}$ in $C$ such that $\Delta-\lim _{n \rightarrow \infty} x_{n}=x$ and $\lim _{n \rightarrow \infty} \operatorname{dist}\left(x_{n}, T\left(x_{n}\right)\right)=$ 0 , one has $x \in T(x)$. Now, we prove the demiclosed principle for Osilike-Berinde- $G$ nonexpansive mappings in complete uniformly convex hyperbolic spaces. Notice that this is an extension of Theorem 4.1 in [17]. 
Theorem 3.2. Let $C$ be a nonempty closed convex subset of $X$ and $G=(V(G), E(G))$ be a directed graph such that $V(G)=C$ and $C$ has property $G$. Let $T: C \rightarrow K(C)$ be an OsilikeBerinde-G-nonexpansive mapping with $L \geq 0$. Then $I-T$ is demiclosed.

Proof. Let $\left\{x_{n}\right\}$ be a sequence in $C$ such that $\Delta-\lim _{n \rightarrow \infty} x_{n}=x$ and $\lim _{n \rightarrow \infty} \operatorname{dist}\left(x_{n}, T\left(x_{n}\right)\right)=0$. For each $n \in \mathbb{N}$, we can choose $y_{n} \in T\left(x_{n}\right)$ and $z_{n} \in T(x)$ such that

$$
d\left(x_{n}, y_{n}\right)=\operatorname{dist}\left(x_{n}, T\left(x_{n}\right)\right) \text { and } d\left(y_{n}, z_{n}\right)=\operatorname{dist}\left(y_{n}, T(x)\right) \text {. }
$$

Since $C$ has property $G$, there exists a subsequence $\left\{x_{n_{k}}\right\}$ of $\left\{x_{n}\right\}$ such that $\left(x_{n_{k}}, x\right) \in$ $E(G)$ for all $k \in \mathbb{N}$. Since $T(x)$ is compact, without loss of generality, we may assume that $\lim _{k \rightarrow \infty} z_{n_{k}}=z \in T(x)$. Since $T$ is Osilike-Berinde- $G$-nonexpansive,

$$
\begin{aligned}
d\left(x_{n_{k}}, z\right) & \leq d\left(x_{n_{k}}, y_{n_{k}}\right)+d\left(y_{n_{k}}, z_{n_{k}}\right)+d\left(z_{n_{k}}, z\right) \\
& \leq d\left(x_{n_{k}}, y_{n_{k}}\right)+H\left(T\left(x_{n_{k}}\right), T(x)\right)+d\left(z_{n_{k}}, z\right) \\
& \leq(1+L) \operatorname{dist}\left(x_{n_{k}}, T\left(x_{n_{k}}\right)\right)+d\left(x_{n_{k}}, x\right)+d\left(z_{n_{k}}, z\right) .
\end{aligned}
$$

This implies that $\limsup d\left(x_{n_{k}}, z\right) \leq \limsup d\left(x_{n_{k}}, x\right)$. Therefore, $z \in A\left(C,\left\{x_{n_{k}}\right\}\right)=\{x\}$ and hence $x=z \in \stackrel{k \rightarrow \infty}{T(x)}$. This shows that $I-T$ is demiclosed.

As a consequence of Theorem 3.2, we can obtain the following fixed point theorem. Notice that it is an extension of Theorem 4.2 in [17].

Theorem 3.3. Let $C$ be a nonempty bounded closed convex subset of $X$ and $G=(V(G), E(G))$ be a convex directed graph such that $V(G)=C$ and $C$ has property $G$. Let $T: C \rightarrow K(C)$ be a Osilike-Berinde-G-nonexpansive mapping. Suppose there exist $u \in C_{T}$ and $\mu \geq 0$ such that

$$
H(T(x), T(y)) \leq d(x, y)+\mu \cdot \operatorname{dist}(y, \alpha u \oplus(1-\alpha) T(x)),
$$

for all $\alpha \in[0,1]$ and $(x, y) \in E(G)$. Then $T$ has a fixed point in $C$.

Proof. For each $n \in \mathbb{N}$, we define $T_{n}: C \rightarrow K(C)$ by

$$
T_{n}(x):=\frac{1}{n} u \oplus\left(1-\frac{1}{n}\right) T(x), \text { for all } x \in C .
$$

Since $T$ is edge-preserving, by the convexity of $G$ we can see that $T_{n}$ is also edge-preserving. Since $u \in C_{T}$, again by the convexity of $G$ we can show that $u \in C_{T_{n}}$ and hence $C_{T_{n}} \neq \emptyset$. It follows from (W4) and (3.4) that

$$
\begin{aligned}
H\left(T_{n}(x), T_{n}(y)\right) & \leq\left(1-\frac{1}{n}\right) H(T(x), T(y)) \\
& \leq\left(1-\frac{1}{n}\right) d(x, y)+\left(1-\frac{1}{n}\right) \mu \cdot \operatorname{dist}\left(y, T_{n}(x)\right),
\end{aligned}
$$

for all $(x, y) \in E(G)$. By Theorem 2.1, there exists $x_{n} \in C$ such that $x_{n} \in T_{n}\left(x_{n}\right)$. Let $y_{n} \in T\left(x_{n}\right)$ be such that

$$
x_{n}=\frac{1}{n} u \oplus\left(1-\frac{1}{n}\right) y_{n} .
$$

Since $\left\{x_{n}\right\}$ is bounded, there exists a subsequence $\left\{x_{n_{k}}\right\}$ of $\left\{x_{n}\right\}$ such that $\Delta-\lim _{k \rightarrow \infty} x_{n_{k}}=$ $x \in C$. Notice also that

$$
\operatorname{dist}\left(x_{n_{k}}, T\left(x_{n_{k}}\right)\right) \leq d\left(x_{n_{k}}, y_{n_{k}}\right)=\frac{1}{n_{k}} d\left(u, y_{n_{k}}\right) \rightarrow 0 \text { as } k \rightarrow \infty \text {. }
$$

By Theorem 3.2, $x \in T(x)$ and this completes the proof.

The condition (3.4) in Theorem 3.3 seems to be strong, but the following example shows that it is a necessary condition. 
Example 3.5. Let $X=(\mathbb{R},|\cdot|), C=[0,1]$ and $G=(V(G), E(G))$ be such that $V(G)=C$ and $E(G)=\{(x, y): x, y \in V(G)\}$. It is easy to see that $G$ is convex and $C$ has property $G$. Let $T: C \rightarrow K(C)$ be defined by

$$
T(x):= \begin{cases}{\left[\frac{2}{3}, 1\right]} & \text { if } x \in\left[0, \frac{1}{2}\right] \\ {\left[0, \frac{1}{3}\right]} & \text { if } x \in\left(\frac{1}{2}, 1\right] .\end{cases}
$$

Let $L=4$ and $x, y \in C$. Without loss of generality, we may assume that $x \in\left[0, \frac{1}{2}\right]$ and $y \in\left(\frac{1}{2}, 1\right]$. Thus, $T(x)=\left[\frac{2}{3}, 1\right]$ and $T(y)=\left[0, \frac{1}{3}\right]$. This implies that $\operatorname{dist}(x, T(x)) \geq \frac{1}{6}$ and $\operatorname{dist}(y, T(y)) \geq \frac{1}{6}$. Hence,

$$
H(T(x), T(y))=H\left(\left[\frac{2}{3}, 1\right],\left[0, \frac{1}{3}\right]\right)=\frac{2}{3} \leq|x-y|+L \cdot \operatorname{dist}(x, T(x))
$$

and

$$
H(T(x), T(y))=H\left(\left[\frac{2}{3}, 1\right],\left[0, \frac{1}{3}\right]\right)=\frac{2}{3} \leq|x-y|+L \cdot \operatorname{dist}(y, T(y)) .
$$

This shows that $T$ is Osilike-Berinde-nonexpansive, and hence Osilike-Berinde- $G$-nonexpansive. Next, we show that $T$ does not satisfy (3.4). Given $u \in C_{T}$ and $\mu \geq 0$. Choose $x=\frac{1}{2}, y=1$ and $\alpha=0$. Then $(x, y) \in E(G), T(x)=\left[\frac{2}{3}, 1\right], T(y)=\left[0, \frac{1}{3}\right]$, and

$$
\operatorname{dist}(y, \alpha u \oplus(1-\alpha) T(x))=\operatorname{dist}(y, T(x))=0 .
$$

This implies that

$$
H(T(x), T(y))=H\left(\left[\frac{2}{3}, 1\right],\left[0, \frac{1}{3}\right]\right)=\frac{2}{3}>\frac{1}{2}=|x-y|+\mu \cdot \operatorname{dist}(y, \alpha u \oplus(1-\alpha) T(x)) .
$$

Obviously, $T$ does not have a fixed point.

\section{CONVERGENCE THEOREMS}

In this section, we prove strong and $\Delta$-convergence theorems of the Ishikawa iteration process for $G$-quasinonexpansive mappings and obtain the results for Osilike-Berinde- $G$ nonexpansive mappings as corollaries. Let $C$ be a nonempty convex subset of $X$, and $\left\{\alpha_{n}\right\},\left\{\beta_{n}\right\}$ be sequences in $[0,1]$, and $T: C \rightarrow C B(C)$ be a multi-valued mapping. The sequence of Ishikawa iteration [31] is defined by $x_{1} \in C$,

$$
y_{n}=\left(1-\beta_{n}\right) x_{n} \oplus \beta_{n} z_{n}, \quad n \in \mathbb{N},
$$

where $z_{n} \in T\left(x_{n}\right)$, and

$$
x_{n+1}=\left(1-\alpha_{n}\right) x_{n} \oplus \alpha_{n} z_{n}^{\prime}, \quad n \in \mathbb{N},
$$

where $z_{n}^{\prime} \in T\left(y_{n}\right)$.

The following lemma is crucial.

Lemma 4.2. Let $C$ be a nonempty convex subset of $X$ and $G=(V(G), E(G))$ be a convex directed graph such that $V(G) \subseteq C$. Let $T: C \rightarrow C B(C)$ be an edge-preserving mapping. Let $\left\{y_{n}\right\}$ and $\left\{x_{n}\right\}$ be defined by (4.5) and (4.6), respectively. If $x_{1}$ dominates $p \in F(T)$, then $x_{n}$ and $y_{n}$ dominate $p$ for all $n \in \mathbb{N}$.

Proof. Since $\left(x_{1}, p\right) \in E(G)$ and $T$ is edge-preserving, $\left(z_{1}, p\right) \in E(G)$. It follows from the convexity of $G$ that $\left(y_{1}, p\right) \in E(G)$. Since $T$ is edge-preserving, $\left(z_{1}^{\prime}, p\right) \in E(G)$. By the convexity of $G$ we have $\left(x_{2}, p\right) \in E(G)$. Continue in this way, we can show that $\left(y_{n}, p\right) \in$ $E(G)$ and $\left(x_{n}, p\right) \in E(G)$ for all $n \geq 2$. 
Recall that a multi-valued mapping $T: C \rightarrow C B(C)$ is said to satisfy the endpoint condition [30] if $F(T) \neq \emptyset$ and $T(x)=\{x\}$ for all $x \in F(T)$. A sequence $\left\{x_{n}\right\}$ in $X$ is said to be Fejér monotone with respect to $C$ if

$$
d\left(x_{n+1}, c\right) \leq d\left(x_{n}, c\right) \text { for all } c \in C \text { and } n \in \mathbb{N} .
$$

The following lemma shows that the sequence of Ishikawa iteration defined by (4.6) is Fejér monotone with respect to the fixed point set of $G$-quasinonexpansive mapping.

Lemma 4.3. Let $C$ be a nonempty convex subset of $X$ and $G=(V(G), E(G))$ be a convex directed graph such that $V(G) \subseteq C$. Let $T: C \rightarrow C B(C)$ be a $G$-quasinonexpansive mapping satisfying the endpoint condition. Let $\left\{x_{n}\right\}$ be defined by (4.6). If $x_{1}$ dominates $F(T)$, then $\left\{x_{n}\right\}$ is Fejér monotone with respect to $F(T)$.

Proof. Let $p \in F(T)$. By Lemma 4.2, $\left\{x_{n}\right\}$ and $\left\{y_{n}\right\}$ dominate $p$. Since $T$ is $G$-quasinonexpansive and satisfies the endpoint condition,

$$
\begin{aligned}
d\left(y_{n}, p\right) & \leq\left(1-\beta_{n}\right) d\left(x_{n}, p\right)+\beta_{n} d\left(z_{n}, p\right) \\
& \leq\left(1-\beta_{n}\right) d\left(x_{n}, p\right)+\beta_{n} H\left(T\left(x_{n}\right), T(p)\right) \\
& \leq d\left(x_{n}, p\right) .
\end{aligned}
$$

This implies that

$$
\begin{aligned}
d\left(x_{n+1}, p\right) & \leq\left(1-\alpha_{n}\right) d\left(x_{n}, p\right)+\alpha_{n} d\left(z_{n}^{\prime}, p\right) \\
& \leq\left(1-\alpha_{n}\right) d\left(x_{n}, p\right)+\alpha_{n} H\left(T\left(y_{n}\right), T(p)\right) \\
& \leq\left(1-\alpha_{n}\right) d\left(x_{n}, p\right)+\alpha_{n} d\left(y_{n}, p\right) \\
& \leq d\left(x_{n}, p\right)
\end{aligned}
$$

Therefore, $\left\{x_{n}\right\}$ is Fejér monotone with respect to $F(T)$.

The following lemmas are also needed.

Lemma 4.4. Let $C$ be a nonempty closed convex subset of $X$ and $T: C \rightarrow C B(C)$ be a multivalued mapping. If $I-T$ is demiclosed, then $F(T)$ is closed in $X$.

Proof. Let $\left\{x_{n}\right\}$ be a sequence in $F(T)$ such that $\lim _{n \rightarrow \infty} x_{n}=x$. Then $\operatorname{dist}\left(x_{n}, T\left(x_{n}\right)\right)=0$ for all $n \in \mathbb{N}$. It follows from the demiclosedness of $I-T$ that $x \in T(x)$, and hence $x \in F(T)$. This shows that $F(T)$ is closed in $X$.

Lemma 4.5. Let $C$ be a nonempty closed convex subset of $X$ and $T: C \rightarrow C B(C)$ be a multivalued mapping such that $I-T$ is demiclosed. If $\left\{x_{n}\right\}$ is a bounded sequence in $C$ such that $\lim _{n \rightarrow \infty} \operatorname{dist}\left(x_{n}, T\left(x_{n}\right)\right)=0$ and $\left\{d\left(x_{n}, v\right)\right\}$ converges for all $v \in F(T)$, then $\omega_{w}\left(x_{n}\right) \subseteq F(T)$. Here $\omega_{w}\left(x_{n}\right):=\bigcup A\left(C,\left\{u_{n}\right\}\right)$, where the union is taken over all subsequences $\left\{u_{n}\right\}$ of $\left\{x_{n}\right\}$. Moreover, $\omega_{w}\left(x_{n}\right)$ consists of exactly one point.

Proof. Let $u \in \omega_{w}\left(x_{n}\right)$, then there exists a subsequence $\left\{u_{n}\right\}$ of $\left\{x_{n}\right\}$ such that $A\left(C,\left\{u_{n}\right\}\right)=$ $\{u\}$. Since $\left\{u_{n}\right\}$ is bounded, there exists a subsequence $\left\{v_{n}\right\}$ of $\left\{u_{n}\right\}$ such that $\Delta-\lim _{n \rightarrow \infty} v_{n}=$ $v \in C$. It follows from Lemma 2.1 and the demiclosedness of $I-T$ that $u=v \in F(T)$. This implies $\omega_{w}\left(x_{n}\right) \subseteq F(T)$. Next, we show that $\omega_{w}\left(x_{n}\right)$ consists of exactly one point. Let $\left\{u_{n}\right\}$ be a subsequence of $\left\{x_{n}\right\}$ with $A\left(C,\left\{u_{n}\right\}\right)=\{u\}$ and let $A\left(C,\left\{x_{n}\right\}\right)=\{x\}$. Since $u \in \omega_{w}\left(x_{n}\right) \subseteq F(T),\left\{d\left(x_{n}, u\right)\right\}$ converges. By Lemma 2.1, $x=u$. This completes the proof.

Now, we prove $\Delta$-convergence theorem. 
Theorem 4.4. Let $C$ be a nonempty closed convex subset of $X$ and $G=(V(G), E(G))$ be a convex directed graph such that $V(G) \subseteq C$. Let $T: C \rightarrow C B(C)$ be a $G$-quasinonexpansive mapping satisfying the endpoint condition and $I-T$ is demiclosed. Let $\alpha_{n}, \beta_{n} \in[a, b] \subset(0,1)$ and $\left\{x_{n}\right\}$ be defined by (4.6) such that $x_{1}$ dominates $F(T)$. Then $\left\{x_{n}\right\} \Delta$-converges to a fixed point of $T$.

Proof. Let $p \in F(T)$. It follows from (2.3) that

$$
\begin{aligned}
d^{2}\left(y_{n}, p\right) & \leq\left(1-\beta_{n}\right) d^{2}\left(x_{n}, p\right)+\beta_{n} d^{2}\left(z_{n}, p\right)-4 c_{M} \beta_{n}\left(1-\beta_{n}\right) d^{2}\left(x_{n}, z_{n}\right) \\
& \leq\left(1-\beta_{n}\right) d^{2}\left(x_{n}, p\right)+\beta_{n} H^{2}\left(T\left(x_{n}\right), T(p)\right)-4 c_{M} \beta_{n}\left(1-\beta_{n}\right) d^{2}\left(x_{n}, z_{n}\right) \\
& \leq d^{2}\left(x_{n}, p\right)-4 c_{M} \beta_{n}\left(1-\beta_{n}\right) d^{2}\left(x_{n}, z_{n}\right) .
\end{aligned}
$$

This implies

$$
\begin{aligned}
d^{2}\left(x_{n+1}, p\right) & \leq\left(1-\alpha_{n}\right) d^{2}\left(x_{n}, p\right)+\alpha_{n} d^{2}\left(z_{n}^{\prime}, p\right)-4 c_{M} \alpha_{n}\left(1-\alpha_{n}\right) d^{2}\left(x_{n}, z_{n}^{\prime}\right) \\
& \leq\left(1-\alpha_{n}\right) d^{2}\left(x_{n}, p\right)+\alpha_{n} H^{2}\left(T\left(y_{n}\right), T(p)\right)-4 c_{M} \alpha_{n}\left(1-\alpha_{n}\right) d^{2}\left(x_{n}, z_{n}^{\prime}\right) \\
& \leq\left(1-\alpha_{n}\right) d^{2}\left(x_{n}, p\right)+\alpha_{n} d^{2}\left(y_{n}, p\right) \\
& \leq d^{2}\left(x_{n}, p\right)-4 c_{M} \alpha_{n} \beta_{n}\left(1-\beta_{n}\right) d^{2}\left(x_{n}, z_{n}\right) .
\end{aligned}
$$

Thus

$$
\sum_{n=1}^{\infty} a^{2}(1-b) d^{2}\left(x_{n}, z_{n}\right) \leq \sum_{n=1}^{\infty} \alpha_{n} \beta_{n}\left(1-\beta_{n}\right) d^{2}\left(x_{n}, z_{n}\right)<\infty .
$$

So that $\lim _{n \rightarrow \infty} d^{2}\left(x_{n}, z_{n}\right)=0$, and hence $\lim _{n \rightarrow \infty} \operatorname{dist}\left(x_{n}, T\left(x_{n}\right)\right)=0$. By Lemma 4.3, $\left\{d\left(x_{n}, v\right)\right\}$ converges for all $v \in F(T)$. By Lemma 4.5, $\omega_{w}\left(x_{n}\right)$ consists of exactly one point and is contained in $F(T)$. This shows that $\left\{x_{n}\right\} \Delta$-converges to an element of $F(T)$.

As a consequence of Theorems 3.2 and 4.4, we can obtain the following corollary.

Corollary 4.1. Let $C$ be a nonempty closed convex subset of $X$ and $G=(V(G), E(G))$ be a convex directed graph such that $V(G)=C$ and $C$ has property $G$. Let $T: C \rightarrow K(C)$ be an Osilike-Berinde-G-nonexpansive mapping satisfying the endpoint condition. Let $\alpha_{n}, \beta_{n} \in[a, b] \subset$ $(0,1)$ and $\left\{x_{n}\right\}$ be defined by (4.6) such that $x_{1}$ dominates $F(T)$. Then $\left\{x_{n}\right\} \Delta$-converges to a fixed point of $T$.

Next, we will prove strong convergence theorems. Recall that a multi-valued mapping $T: C \rightarrow C B(C)$ is said to satisfy condition (IG) if $F(T) \neq \emptyset$ and there exists a nondecreasing function $f:[0, \infty) \rightarrow[0, \infty)$ with $f(0)=0, f(r)>0$ for $r \in(0, \infty)$ such that $\operatorname{dist}(x, T(x)) \geq f(\operatorname{dist}(x, F(T)))$ for all $x$ which dominates $F(T)$. The following fact can be found in [8].

Lemma 4.6. Let $E$ be a nonempty closed subset of $X$ and $\left\{x_{n}\right\}$ a Fejér monotone sequence with respect to $E$. Then $\left\{x_{n}\right\}$ converges strongly to an element of $E$ if and only if $\lim _{n \rightarrow \infty} \operatorname{dist}\left(x_{n}, E\right)=0$.

Theorem 4.5. Let $C$ be a nonempty closed convex subset of $X$ and $G=(V(G), E(G))$ be a convex directed graph such that $V(G) \subseteq C$. Let $T: C \rightarrow C B(C)$ be a $G$-quasinonexpansive mapping satisfying the endpoint condition. Suppose that $T$ satisfies condition (IG) and $I-T$ is demiclosed. Let $\alpha_{n}, \beta_{n} \in[a, b] \subset(0,1)$ and $\left\{x_{n}\right\}$ be defined by (4.6) such that $x_{1}$ dominates $F(T)$. Then $\left\{x_{n}\right\}$ converges strongly to a fixed point of $T$.

Proof. By Lemma 4.4, $F(T)$ is closed in $X$. As in the proof of Theorem 4.4, we can show that $\lim _{n \rightarrow \infty} \operatorname{dist}\left(x_{n}, T\left(x_{n}\right)\right)=0$. Since $T$ satisfies condition (IG), $\lim _{n \rightarrow \infty} \operatorname{dist}\left(x_{n}, F(T)\right)=0$. By Lemma 4.3, $\left\{x_{n}\right\}$ is Fejér monotone with respect to $F(T)$. The conclusion follows from Lemma 4.6. 
Corollary 4.2. Let $C$ be a nonempty closed convex subset of $X$ and $G=(V(G), E(G))$ be a convex directed graph such that $V(G) \subseteq C$. Let $T: C \rightarrow C B(C)$ be an Osilike-Berinde- $G$ nonexpansive mapping satisfying the endpoint condition. Suppose that $T$ satisfies condition (IG). Let $\alpha_{n}, \beta_{n} \in[a, b] \subset(0,1)$ and $\left\{x_{n}\right\}$ be defined by (4.6) such that $x_{1}$ dominates $F(T)$. Then $\left\{x_{n}\right\}$ converges strongly to a fixed point of $T$.

The following example supports Theorem 4.4.

Example 4.6. Let $X=(\mathbb{R},|\cdot|), C=[0,1]$ and $G=(V(G), E(G))$ be such that $V(G)=[0,1)$ and $E(G)=\{(x, y): x, y \in V(G)\}$. Let $T: C \rightarrow C B(C)$ be defined by

$$
T(x)= \begin{cases}{\left[0, x^{2}\right]} & \text { if } x \in[0,1) \\ \{1\} & \text { if } x=1 .\end{cases}
$$

It is easy to see that $G$ is convex and $T$ is edge-preserving. Notice also that $F(T)=\{0,1\}$ and $T$ satisfies the endpoint condition. If $x=1$ and $y=\frac{1}{2}$, then

$$
H(T(x), T(y))=H\left(\{1\},\left[0, \frac{1}{4}\right]\right)=1>\frac{1}{2}=|x-y| .
$$

This implies that $T$ is not quasinonexpansive. On the other hand, if $(x, y) \in E(G)$ such that $y \in F(T)$, then $y=0$ and hence

$$
H(T(x), T(y))=H\left(\left[0, x^{2}\right],\{0\}\right)=x^{2} \leq x=|x-y| .
$$

This shows that $T$ is $G$-quasinonexpansive. Moreover, if $\left\{v_{n}\right\}$ is a sequence in $C$ such that $\Delta-\lim _{n \rightarrow \infty} v_{n}=v$ and $\lim _{n \rightarrow \infty} \operatorname{dist}\left(v_{n}, T\left(v_{n}\right)\right)=0$, then either $v=0$ or $v=1$. This implies that $I-T$ is demiclosed. Let $\alpha_{n}, \beta_{n} \in[a, b] \subset(0,1)$. By Theorem 4.4, for any starting point $x_{1}$ in $[0,1)$, the sequence $\left\{x_{n}\right\}$ defined by (4.6) converges to a point $x$ in $F(T)$. However, since $1>x_{1} \geq x_{2} \geq \ldots$, it must be the case that $x=0$.

Finally, we prove a strong convergence theorem for hemicompact mappings. Recall that a multi-valued mapping $T: C \rightarrow C B(C)$ is said to be hemicompact if for any sequence $\left\{x_{n}\right\}$ in $C$ such that $\lim _{n \rightarrow \infty} \operatorname{dist}\left(x_{n}, T\left(x_{n}\right)\right)=0$, there exists a subsequence $\left\{x_{n_{k}}\right\}$ of $\left\{x_{n}\right\}$ and $q \in C$ such that $\lim _{k \rightarrow \infty} x_{n_{k}}=q$. The following fact is also needed.

Lemma 4.7. ([24]) Let $\left\{\alpha_{n}\right\},\left\{\beta_{n}\right\}$ be two real sequences in $[0,1)$ such that $\beta_{n} \rightarrow 0$ and $\sum \alpha_{n} \beta_{n}=$ $\infty$. Let $\left\{\gamma_{n}\right\}$ be a nonnegative real sequence such that $\sum \alpha_{n} \beta_{n}\left(1-\beta_{n}\right) \gamma_{n}<\infty$. Then $\left\{\gamma_{n}\right\}$ has a subsequence which converges to zero.

Theorem 4.6. Let $C$ be a nonempty closed convex subset of $X$ and $G=(V(G), E(G))$ be a convex directed graph such that $V(G) \subseteq C$ and $C$ has property $G$. Let $T: C \rightarrow C B(C)$ be an Osilike-Berinde-G-nonexpansive mapping satisfying the endpoint condition. Let $\alpha_{n}, \beta_{n} \in[0,1)$ be such that $\beta_{n} \rightarrow 0$ and $\sum \alpha_{n} \beta_{n}=\infty$ and $\left\{x_{n}\right\}$ be defined by (4.6) such that $x_{1}$ dominates $F(T)$. If $T$ is hemicompact, then $\left\{x_{n}\right\}$ converges strongly to a fixed point of $T$.

Proof. From (4.7) we get that

$$
\sum_{n=1}^{\infty} \alpha_{n} \beta_{n}\left(1-\beta_{n}\right) d^{2}\left(x_{n}, z_{n}\right)<\infty .
$$

By Lemma 4.7, there exist subsequences $\left\{x_{n_{k}}\right\}$ and $\left\{z_{n_{k}}\right\}$ of $\left\{x_{n}\right\}$ and $\left\{z_{n}\right\}$ respectively, such that $\lim _{k \rightarrow \infty} d\left(x_{n_{k}}, z_{n_{k}}\right)=0$, and hence $\lim _{k \rightarrow \infty} \operatorname{dist}\left(x_{n_{k}}, T\left(x_{n_{k}}\right)\right)=0$. Since $T$ is hemicompact and $C$ has property $G$, by passing to a subsequence, we may assume that there exists 
$q \in C$ such that $\lim _{k \rightarrow \infty} x_{n_{k}}=q$ and $\left(x_{n_{k}}, q\right) \in E(G)$ for all $k \in \mathbb{N}$. Since $T$ is Osilike-Berinde$G$-nonexpansive, there exists $L \geq 0$ such that

$$
H\left(T\left(x_{n_{k}}\right), T(q)\right) \leq d\left(x_{n_{k}}, q\right)+L \cdot \operatorname{dist}\left(x_{n_{k}}, T\left(x_{n_{k}}\right)\right) \text { for all } k \in \mathbb{N} .
$$

This implies that

$$
\begin{aligned}
\operatorname{dist}(q, T(q)) & \leq d\left(q, x_{n_{k}}\right)+\operatorname{dist}\left(x_{n_{k}}, T\left(x_{n_{k}}\right)\right)+H\left(T\left(x_{n_{k}}\right), T(q)\right) \\
& \leq 2 d\left(x_{n_{k}}, q\right)+(1+L) \operatorname{dist}\left(x_{n_{k}}, T\left(x_{n_{k}}\right)\right) \rightarrow 0 \text { as } k \rightarrow \infty .
\end{aligned}
$$

Thus $q \in T(q)$. By Lemma 4.3, $\lim _{n \rightarrow \infty} d\left(x_{n}, q\right)$ exists and hence $q$ is the strong limit of $\left\{x_{n}\right\}$.

Acknowledgements. The authors would like to thank the anonymous reviewers for their careful reading and valuable suggestions which led to the present form of the paper. This research was supported by Chiang Mai University and National Research Council of Thailand.

\section{REFERENCES}

[1] Alfuraidan, M. R. and Khamsi, M. A., Fixed points of monotone nonexpansive mappings on a hyperbolic metric space with a graph, Fixed Point Theory Appl., 2015:44 (2015), 1-10

[2] Balog, L. and Berinde, V., Fixed point theorems for nonself Kannan type contractions in Banach spaces endowed with a graph, Carpathian J. Math., 32 (2016), 293-302

[3] Beg, I., Butt, A. R. and Radojević, S., The contraction principle for set valued mappings on a metric space with a graph, Comput. Math. Appl., 60 (2010), 1214-1219

[4] Berinde, M. and Berinde, V., On a general class of multi-valued weakly Picard mappings, J. Math. Anal. Appl., 326 (2007), 772-782

[5] Berinde, V. and Păcurar, M., The contraction principle for nonself mappings on Banach spaces endowed with a graph, J. Nonlinear Convex Anal., 16 (2015), 1925-1936

[6] Bunlue, N. and Suantai, S., Existence and convergence theorems for Berinde nonexpansive multivalued mapping on Banach spaces, Afrika Mat., 30 (2019), 483-494

[7] Chifu, C., Karapınar, E. and Petrussel, G., Qualitative properties of the solution of a system of operator inclusions in b-metric spaces endowed with a graph, Bull. Iranian Math. Soc., 44 (2018), 1267-1281

[8] Chuadchawna, P., Farajzadeh, A. and Kaewcharoen, A., Convergence theorems and approximating endpoints for multivalued Suzuki mappings in hyperbolic spaces, J. Comp. Anal. Appl., 28 (2020), 903-916

[9] Dhompongsa, S. and Panyanak, B., On $\Delta$-convergence theorems in CAT(0) spaces, Comput. Math. Appl., 56 (2008), 2572-2579

[10] Dinevari, T. and Frigon, M., Fixed point results for multivalued contractions on a metric space with a graph, J. Math. Anal. Appl., 405 (2013), 507-517

[11] Edelstein, M., On fixed and periodic points under contractive mappings, J. London Math. Soc., 37 (1962), 74-79

[12] Geraghty, M. A., On contractive mappings, Proc. Amer. Math. Soc., 40 (1973), 604-608

[13] Hussain, N., Berinde, V. and Shafqat, N., Common fixed point and approximation results for generalized $\phi-$ contractions, Fixed Point Theory, 10 (2009), 111-124

[14] Jachymski, J., The contraction principle for mappings on a metric space with a graph, Proc. Amer. Math. Soc., 136 (2008), 1359-1373

[15] Khamsi, M. A. and Khan, A. R., Inequalities in metric spaces with applications, Nonlinear Anal., 74 (2011), 4036-4045

[16] Kirk, W. A. and Panyanak, B., A concept of convergence in geodesic spaces, Nonlinear Anal. 68 (2008), 3689-3696

[17] Klangpraphan, C. and Panyanak, B., Fixed point theorems for some generalized multi-valued nonexpansive mappings in Hadamard spaces, Thai J. Math., 17 (2019), 543-555

[18] Laokul, T. and Panyanak, B., A generalization of the (CN) inequality and its applications, Carpathian J. Math., 36 (2020), 81-90

[19] Leuştean, L., A quadratic rate of asymptotic regularity for CAT(0)-spaces, J. Math. Anal. Appl., 325 (2007), 386-399

[20] Leuştean, L., Nonexpansive iterations in uniformly convex W-hyperbolic spaces, in Nonlinear Analysis and Optimization I. Nonlinear Analysis, vol. 513 of Contemporary Mathematics, pp. 193-210, American Mathematical Society, Providence, RI, USA, 2010 
[21] O'Regan, D. and Petruşel, A., Fixed point theorems for generalized contractions in ordered metric spaces, J. Math. Anal. Appl., 341 (2008), 1241-1252

[22] Osilike, M. O., Stability results for fixed point iteration procedures, J. Nigerian Math. Soc., 14/15 (1995/96), 17-29

[23] Panyanak, B., Fixed points of multivalued $G$-nonexpansive mappings in Hadamard spaces endowed with graphs, J. Funct. Spaces, 2020, Article ID 5849262, (2020), 1-8

[24] Panyanak, B., Mann and Ishikawa iterative processes for multivalued mappings in Banach spaces, Comput. Math. Appl., 54 (2007), 872-877

[25] Panyanak, B., On an open problem of Kyung Soo Kim, Fixed Point Theory Appl., 2015:186 (2015), 1-12

[26] Panyanak, B., The viscosity approximation method for multivalued $G$-nonexpansive mappings in Hadamard spaces endowed with graphs, J. Fixed Point Theory Appl., 22:90 (2020), 1-18

[27] Petruşel, A. and Rus, I. A., Fixed point theorems in ordered L-spaces, Proc. Amer. Math. Soc., 134 (2006), 411-418

[28] Puttasontiphot, T., Mann and Ishikawa iteration schemes for multivalued mappings in CAT(0) spaces, Appl. Math. Sci., 4 (2010), 3005-3018

[29] Rus, I. A., On a general fixed point principle for $(\theta, \phi)$-contractions, Studia Univ. Babeş-Bolyai Math., 34 (1989), 65-70

[30] Shahzad, N. and Markin, J., Invariant approximations for commuting mappings in CAT(0) and hyperconvex spaces, J. Math. Anal. Appl., 337 (2008), 1457-1464

[31] Shahzad, N. and Zegeye, H., On Mann and Ishikawa iteration schemes for multi-valued maps in Banach spaces, Nonlinear Anal., 71 (2009), 838-844

[32] Sridarat, P., Suparaturatorn, R., Suantai, S., Cho, Y. J., Convergence analysis of SP-iteration for G-nonexpansive mappings with directed graphs, Bull. Malays. Math. Sci. Soc. 42 (2019), 2361-2380

[33] Tiammee, J., Kaewkhao, A. and Suantai, S., On Browder's convergence theorem and Halpern iteration process for $G-$ nonexpansive mappings in Hilbert spaces endowed with graphs, Fixed Point Theory Appl., 187 (2015), 1-12

[34] Tiammee, J. and Suantai, S., Coincidence point theorems for graph-preserving multi-valued mappings, Fixed Point Theory Appl., 2014:70 (2014), 1-11

[35] Tripak, O., Common fixed points of G-nonexpansive mappings on Banach spaces with a graph, Fixed Point Theory Appl., 2016:87 (2006), 1-8

[36] Vetro, C. and Vetro, F., Metric or partial metric spaces endowed with a finite number of graphs: a tool to obtain fixed point results, Topology Appl., 164 (2014), 125-137

[37] Xu, H. K., Inequalities in Banach spaces with applications, Nonlinear Anal., 16 (1991), 1127-1138

[38] Yambangwai, D., Aunruean, S. and Thianwan, T., A new modified three-step iteration method for G-nonexpansive mappings in Banach spaces with a graph, Numer. Algorithms, 84 (2020), 537-565

${ }^{1}$ Research Center in Mathematics and Applied Mathematics

DEPARTMENT OF MATHEMATICS

Faculty of SCience, Chiang Mai University, Chiang Mai 50200, Thailand

2 Data Science Research Center

DEPARTMENT OF MATHEMATICS

Faculty OF SCIEnCE, Chiang Mai University, Chiang Mai 50200, Thailand

Email address: bancha.p@cmu.ac.th 